\title{
IncRNA-Associated ceRNA Networks in Spleen of Nocth1-correlated T-ALL Leukemia Mice
}

\section{CURRENT STATUS: POSTED}

Research Square

\section{Min Shi}

Second Hospital of Hebei Medical University

Shaohua Wang

Second Hospital of Hebei Medical University

Xiaofei Li

Second Hospital of Hebei Medical University

Yongjun Li

Second Hospital of Hebei Medical University

sm8344@hotmail.comCorresponding Author

\section{DOI:}

$10.21203 / \mathrm{rs} .2 .21227 / \mathrm{v} 1$

\section{SUBJECT AREAS}

\section{Oncology Cancer Biology}

\section{KEYWORDS}

T-ALL, Nocth1, deep RNA-sequencing analysis, InCRNA, ceRNA 
Abstract

Background

Acute T-lymphocytic leukemia (T-ALL) is a highly aggressive malignant tumor in leukemia. Nocth1 is considered as an major oncogene in the development of T-ALL. Increasing evidences have revealed that the occurrence and progression of T-ALL referred to abnormal gene expression, pathway activation and the regulation between these genes. However, the potential IncRNA-associated competing endogenous RNA (ceRNA) network involved in spleen of Nocth1-correlated T-ALL leukemia mice remains unclear.

Methods

Overexpression of Notch intracellular domain (ICN) of Notch1 by retroviral infection was used to set up mouse T-ALL model. Deep RNA-sequencing analysis was performed the expression of IncRNAs and mRNA in spleen of T-ALL mice and C57BL/6 mice.

Results

The deep RNA-sequencing analysis shown that 1833 IncRNAs and 4626 mRNAs were deregulated according to the P-value $(p<0.05)$ and fold change $(>2$-fold $)$ in spleen of T-ALL leukemia mice compared with that of C57BL/6 mice. Gene Ontology(GO) and KEGG pathway analysis were performed to reveal the potential roles of differentiated expressed IncRNAs. Co-expression Network was performed to reveal the regulation relationship between the differentiated expressed IncRNAs and mRNAs. CeRNA prediction constructed the IncRNA-miRNA-mRNA model to find the core ceRNA based on regression model analysis and seed sequence matching methods.

Conclusion

This study provided a systematic overview of the altered IncRNAs and mRNA expression, pathway and ceRNA regulation network in the pathogenesis of Nocth1-correlated T-ALL.

Full Text

Due to technical limitations, full-text HTML conversion of this manuscript could not be completed. However, the manuscript can be downloaded and accessed as a PDF.

Tables

Table 1 Basic information of selected nice IncRNAs for qRT-PCR confirmation. 


\begin{tabular}{|l|l|l|l|l|l|l|}
\hline Seqname & Source & RNA length & Locus & P-value & Fold change & Regulation \\
\hline NONMMUT117521.1 & PMID24463510 & 863 & $\begin{array}{l}4: 137882371- \\
137883938\end{array}$ & 0.007679055 & 7.33 & DOWN \\
\hline NONMMUT021309.2 & PMID23951020 & 2508 & $\begin{array}{l}14.65114738- \\
65117245\end{array}$ & 0.005231906 & 5.65 & DOWN \\
\hline ENSMUST00000195494 & noncodeV3 & 2099 & $2: 11532596-11534694$ & 0.000178499 & 10.60 & DOWN \\
\hline NONMMUT008951.2 & noncodeV3 & 3828 & $\begin{array}{l}11: 24103379- \\
24107440\end{array}$ & $4.34 \mathrm{E}-05$ & 12.53 & DOWN \\
\hline NONMMUT008779.2 & noncodeV3 & 3379 & $\begin{array}{l}11: 20461259- \\
20464664\end{array}$ & 0.00264931 & 9.67 & DOWN \\
\hline NONMMUT026003.2 & noncodeV3 & 2078 & $\begin{array}{l}16: 23981313- \\
23983390\end{array}$ & 0.004331438 & 5.59 & DOWN \\
\hline NONMMUT041921.2 & NONCODEv4 & 3925 & $\begin{array}{l}2: 181405971- \\
181409895\end{array}$ & 0.000122807 & 7.66 & DOWN \\
\hline NONMMUT147972.1 & - & 1666 & $4: 32239436-32241101$ & 0.001742802 & 8.78 & DOWN \\
\hline NONMMUT029828.2 & NONCODEv3 & 2875 & $\begin{array}{l}17: 46424215- \\
46427089\end{array}$ & $3.45 \mathrm{E}-05$ & 32.22 & DOWN \\
\hline
\end{tabular}

Table 2 Specific primers of each IncRNA.

\begin{tabular}{|l|l|l|}
\hline Seqname & Forward & Reverse \\
\hline NONMMUT117521.1 & GATGCTGAGCAAGTGGGTGA & TGGATAGGTGGATGTGAGCG \\
\hline NONMMUT021309.2 & TCCAACATGCTCACCCGAG & AGCATGGCGCTGTCAATGTA \\
\hline ENSMUST00000195494 & GGATCTGCAGAGGGGCTTAC & GCACCAGGGAACCCATTGTA \\
\hline NONMMUT008951.2 & CAGGACGAGTCAGGCACATT & CAAGCCTTCGGTCTGTGTCT \\
\hline NONMMUT008779.2 & AGTCCTTGTGTGAGCATCCG & CCCATGTGTTCCGTGGAGAA \\
\hline NONMMUT026003.2 & AAGAGGCTGGCTAGACCTGA & AAGGAGTTGAAGGCGGGAG \\
\hline NONMMUT041921.2 & AAGATGTAAGGGCCACCAGC & TGTGAGGCTGTTCCAAGTCC \\
\hline NONMMUT147972.1 & ACCCAACAAGATGCTCCTCG & AAGTCGCAGTTGGGTCTCTG \\
\hline NONMMUT029828.2 & TTCAGGTTCAGCTGTTCGGG & GAGTGGGGAGCCGTAAGAAC \\
\hline
\end{tabular}

Figures 
A
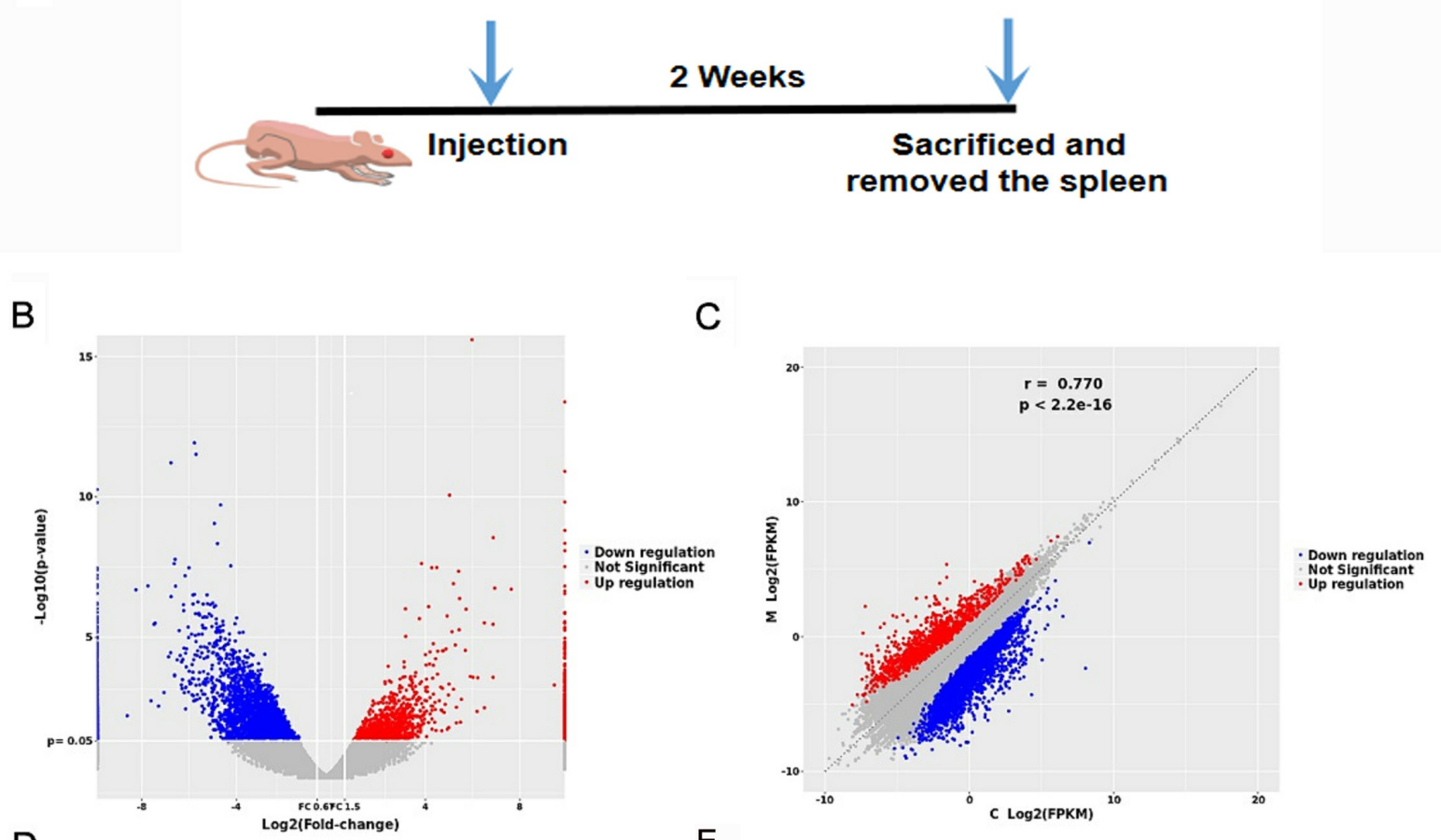

D
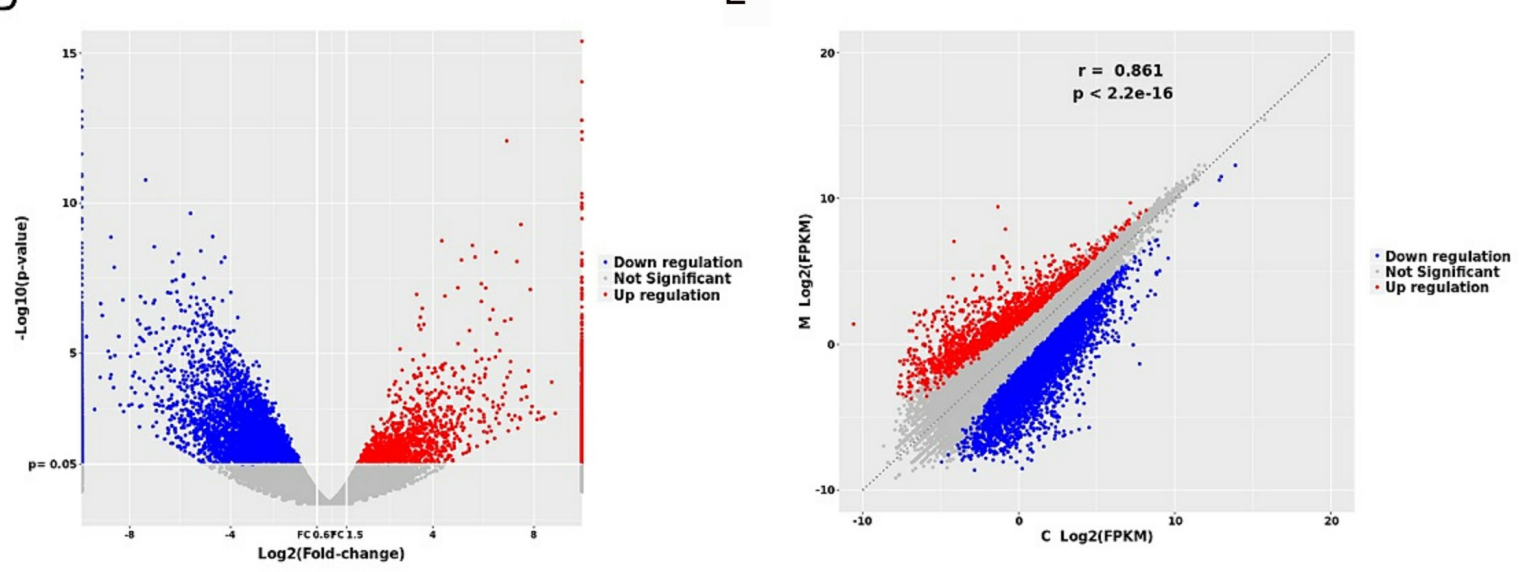

Figure 1

Differentially expressed IncRNAs and mRNAs in spleen of mice. The time-line of study design

(A). Differentially expressed IncRNAs are shown in volcano Plot(B) 16 and scatter-Plot(C).

Differentially expressed mRNAs are shown in volcano plot(D) and scatter plot(E). For volcano plot, the abscissa is fold change(fold change $\geq 2)$ and the ordinate is $p$ value( $p$ value $\leq 0.05)$. The red color is the up-regulated genes and the blue is the down-regulated genes. For scatter plot, The abscissa and ordinate represent two sets of samples. The red color is the up-regulated genes and the blue is the down-regulated genes. 
A

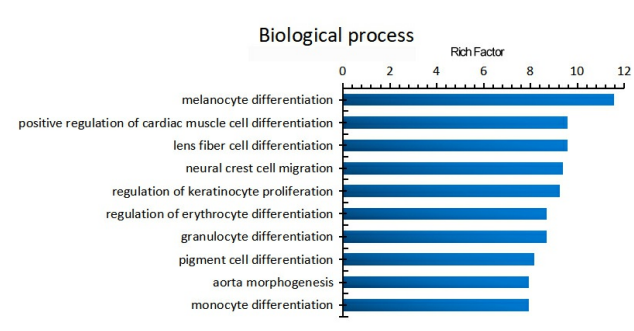

C

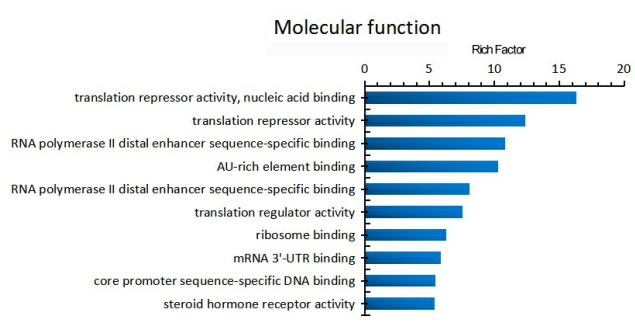

B

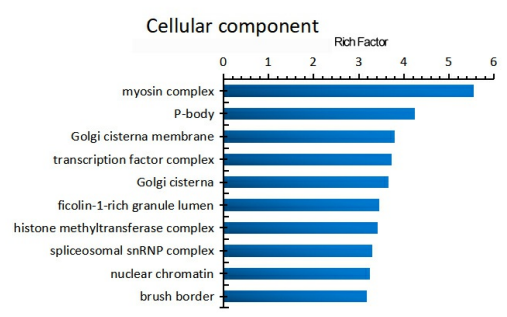

D

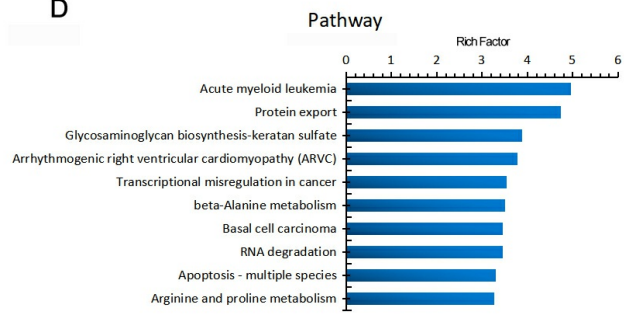

Figure 2

mRNA GO and KEGG pathway analysis in spleen of mice. TOP10 enriched GO terms for

Biological process(A), cellular component(B) and molecular function(C). TOP10 enriched KEGG pathway was shown in corresponded to differentially expressed transcripts(D). 


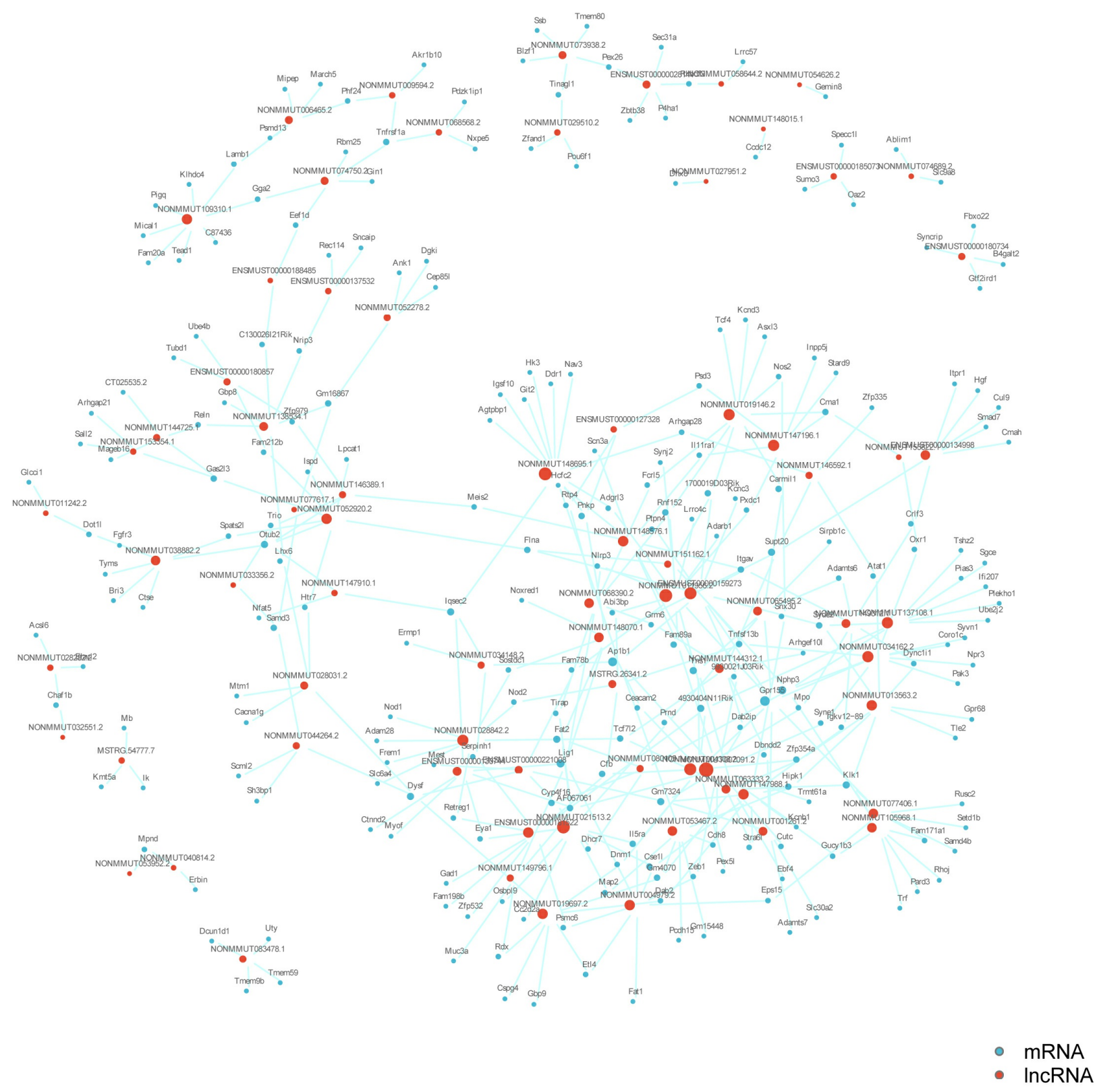

Figure 3

IncRNAs and mRNAs co-expression analysis. This network shown the co-expression correlations between IncRNAs and mRNAs. 


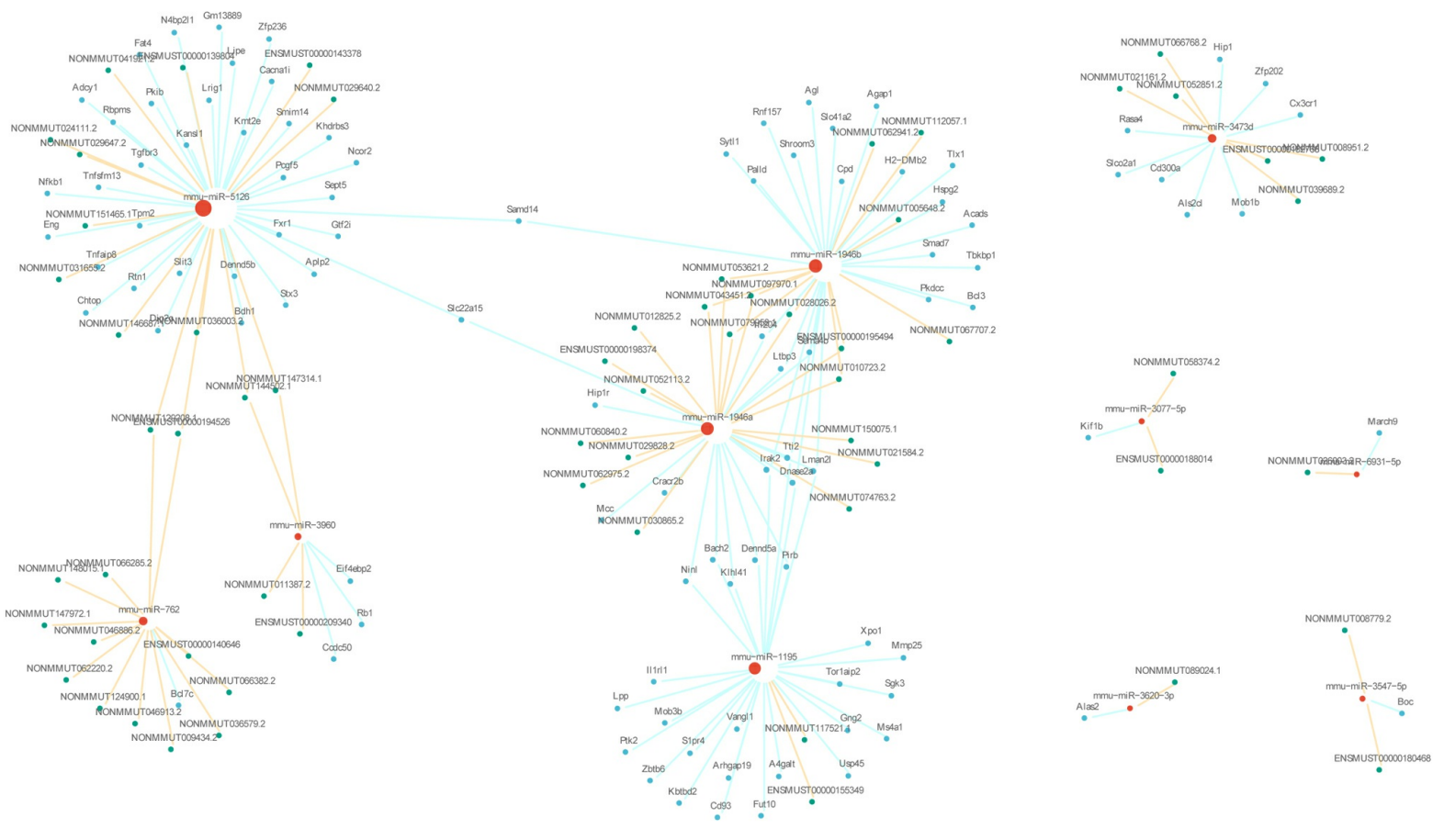

Figure 4

Construction of a ceRNA network. Global view of the ceRNA network. Six networks were presented. 

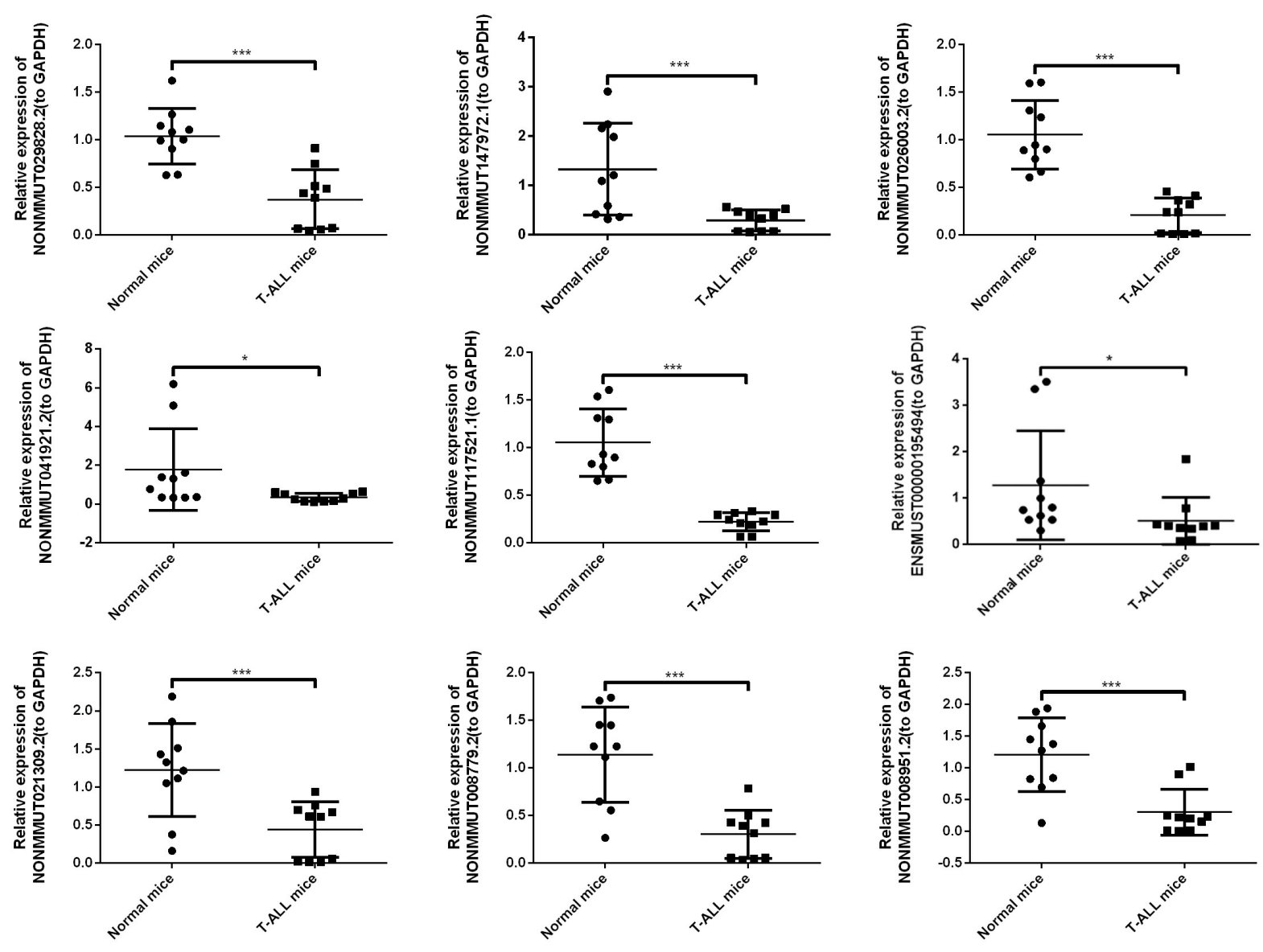

Figure 5

Validation of deep RNA sequence results by qRT-PCR from spleen of T-ALL mice or C57BL/6 mice.

\section{Supplementary Files}

This is a list of supplementary files associated with this preprint. Click to download. additional data.xls

ARRIVE Guidelines Checklist.pdf 